\title{
Characterization of the response of a dual resonance of an arc-induced long-period grating to various physical parameters
}

\author{
Paulo Caldas, ${ }^{1,2,3, *}$ Gaspar Rego, ${ }^{1,3}$ Oleg V. Ivanov, ${ }^{4}$ and José L. Santos ${ }^{1,2}$ \\ ${ }^{1}$ INESC Porto, Rua do Campo Alegre 687, 4169-007 Porto, Portugal \\ ${ }^{2}$ Departamento de Física, Faculdade de Ciências, University of Porto, \\ Rua do Campo Alegre 687, 4169-007 Porto, Portugal \\ ${ }^{3}$ Escola superior de Tecnologia e Gestão, Viana do Castelo, \\ Av. do Atlântico, Apartado 574, 4900-348 Viana do Castelo, Portugal \\ ${ }^{4}$ Ulyanovsk Branch of Kotel'nikov, Institute of Radio Engineering and Electronics, \\ Russian Academy of Sciences, ul. Goncharova 48, Ulyanovsk 432011, Russia \\ ${ }^{*}$ Corresponding author: pcaldas @ inescporto.pt
}

Received 11 December 2009; revised 24 April 2010; accepted 4 May 2010; posted 4 May 2010 (Doc. ID 121363); published 20 May 2010

\begin{abstract}
We present results on the characterization of the response of a dual resonance observed in the spectrum of a single long-period grating arc-induced in a B/Ge co-doped fiber to different physical parameters. The dual resonance is formed by two overlapping resonances corresponding to coupling of the core mode to symmetric and antisymmetric cladding modes. The behavior of the resonances is studied when the grating is subject to strain, bending, torsion, temperature, or external refractive-index changes. The strain, bending, and torsion sensitivities of the two resonances differ, whereas the temperature sensitivities are almost the same. The sensitivities to variation in external refractive index are the same for the two resonances when the long-period grating is straight and differ when the fiber with the grating is curved. (C) 2010 Optical Society of America

OCIS codes: $\quad 280.4788,050.2770$.
\end{abstract}

\section{Introduction}

In the past few years some effort has been made to investigate the mechanisms responsible for the formation of arc-induced gratings. It has been shown [1] that the gratings arc-induced in the SMF-28 Corning fiber couple the core mode to antisymmetric cladding modes whereas the ones produced in the FIBERCORE PS1250/1500 B/Ge co-doped fiber are due to coupling to symmetric modes. Recently, it was demonstrated that the origin of the antisymmetric coupling in the case of standard fiber is the thermal gradient in the arc discharge [2]. This gra-

0003-6935/10/162994-06\$15.00/0

(C) 2010 Optical Society of America dient induces periodic microdeformations in the fiber that form the antisymmetric grating. In the case of B/ Ge co-doped fiber a symmetric mechanism plays the main role [3]. At the same time, it should also be possible to induce antisymmetric microdeformations in that fiber. By moving the fiber from the centerline that joins the electrodes to a region in which the thermal gradient is higher and the average temperature is lower, the formation of the antisymmetric grating was promoted and the formation of the symmetric grating was suppressed. Thus, in a B/Ge co-doped fiber, it was possible to fabricate an arc-induced longperiod grating (LPG) whose spectrum contained a dual set of resonances [3]. These paired resonances are formed by different mechanisms and are caused by coupling to cladding modes of different symme- 
tries. Therefore, we can expect these resonances to respond differently to changes in various physical parameters.

It is known that LPGs have been used as sensor elements that can be used to perform simultaneous measurement of various physical parameters [4]. However, the proposed approaches require, in general, the use of two optical sources or different fiber types [ㅁ,6]. Recently, we demonstrated a step changed grating for simultaneous measurement of temperature and strain, in which the previous drawbacks were overcome [7]. The specific properties of the dual set of resonances opens the possibility to implement a novel compact single-fiber sensor based on LPGs for simultaneous measurement of different physical parameters. Such a sensor would require a single light source for its interrogation, it would also be easier to fabricate, through a one-step process, and would enable discrimination between physical parameters other than temperature and strain.

We characterize the response of the grating with a dual resonance in its spectrum to temperature, strain, bending, torsion, and to variation of the external refractive index.

\section{Temperature and Strain Sensitivities}

For grating fabrication, a setup was used in which a fiber under tension was placed between the electrodes of a splicing machine while the fiber was heated locally by exposure to a sequence of arc discharges [8]. After each discharge, the fiber was moved by the grating period and another discharge was applied. The whole process of fiber displacement and arc discharge was repeated several times under computer control. A white light source and an optical spectrum analyzer (OSA) were used to monitor the evolution of the grating spectrum. To obtain a grating with a double resonance, we positioned the fiber in a region of the arc in which the average temperature was lower and the thermal gradient was higher, and we also applied a higher pulling tension, in comparison with the previous experiments [2]. The fabrication parameters were set as follows: 60 periods of $540 \mu \mathrm{m}$ using a tension of $23 \mathrm{~g}$ and discharges with a current of $9 \mathrm{~mA}$ and duration of $0.5 \mathrm{~s}$. Figure 1 shows two neighboring resonances in the third telecommunication window, Dip1 and Dip2, belonging, respectively, to coupling to antisymmetric and symmetric cladding modes [3].

First, the response of the grating to changes in temperature and strain was investigated. The tem-

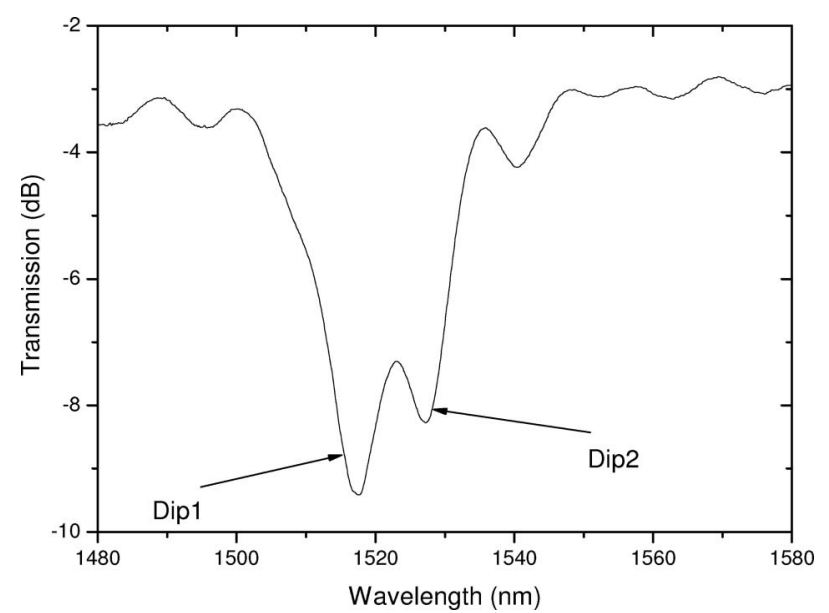

Fig. 1. Grating spectrum.

perature sensitivity of long-period fiber gratings is caused by the thermo-optic effect, which affects the refractive index of the fiber. The resonance wavelengths of a LPG are determined by the difference between propagation constants of the core and cladding modes. These propagation constants change when the fiber temperature increases [9], which results in shifts of the resonance wavelengths.

Figure 2 shows the experimental setup used to study the temperature behavior. The fiber with the LPG inscribed on it was placed inside a tubular oven; the fiber under tension was created by an attached mass of $5.1 \mathrm{~g}$. The temperature was increased in steps of $10^{\circ} \mathrm{C}$ up to $120^{\circ} \mathrm{C}$. As seen in Fig. 3, both resonances shift toward shorter wavelengths with the increase in temperature and temperature sensitivity almost identical $\left(-288 \mathrm{pm} /{ }^{\circ} \mathrm{C}\right)$.

The strain sensitivity of a LPG arises from strainoptic effects and geometric deformation of fiber [9], which modify the propagation constants of the core and cladding modes, the magnitude of change dependent on the mode profile over the fiber cross section. The symmetric and antisymmetric modes have different profiles; therefore, the two corresponding peaks in the spectrum have, in general, different strain sensitivities.

The setup used to characterize the response of the LPG to strain and other parameters is shown in Fig. 4. The fiber with the LPG is mounted between two translation stages. The grating was centered between them. It is possible to apply tension by moving the linear stage away from the fixed support. The LPG was illuminated by a broadband source (with

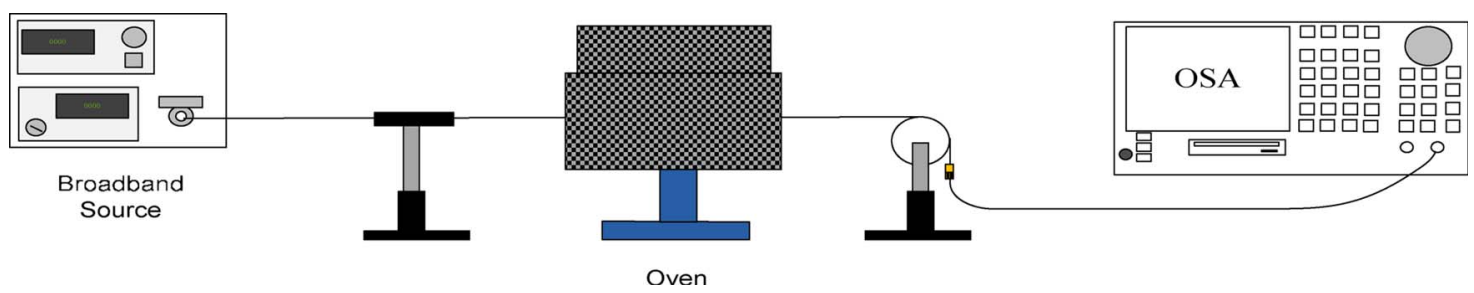

Fig. 2. (Color online) Experimental setup for temperature measurements. 


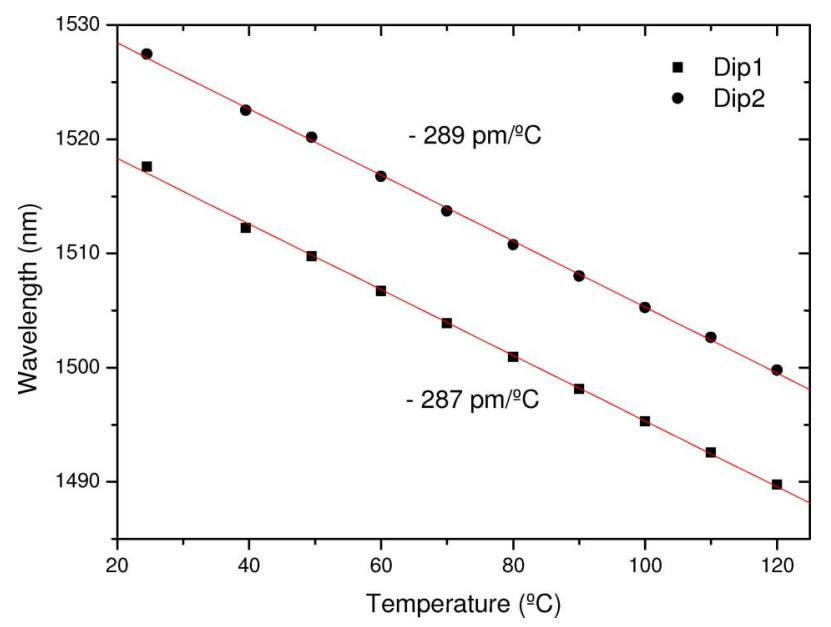

Fig. 3. (Color online) Dependence on temperature of the resonance wavelengths of Dip1 and Dip2.

the center wavelength at $1550 \mathrm{~nm}$ ) and the shift of the resonances is measured by an OSA. For strain sensitivity, the fiber was fixed at two points separated by $30 \mathrm{~cm}$, one of which was positioned on a translation stage. The fiber was then stretched in steps of $167 \mu \varepsilon$ up to $2000 \mu \varepsilon$ and then back again to zero.

The results of strain measurements are shown in Fig. 5 . The sensitivity value of $0.172 \mathrm{pm} / \mu \varepsilon$ obtained for the resonance at longer wavelengths is almost half of the value $(0.366 \mathrm{pm} / \mu \varepsilon)$ for the resonance at shorter wavelengths. As can be seen from the figure, some dispersion of the measurement points from the linear slope can be observed for both dips with stronger dispersion for Dip1 having larger strain sensitivity. This fact cannot be accounted for by the uncertainty of the micrometer positioner. The reason for the dispersion could be related to fluctuations of the optical power supplied by the broadband source or temperature variations, since the LPG is sensitive to changes in temperature, when even light air drifts can compromise the measurements.

\section{Bending, Torsion, and Refractive-Index Sensitivities}

The same setup was used for bending, torsion, and external refractive-index measurements. With this configuration the LPG can be bent when the linear stage is moved toward the fixed support (see Fig. 6). The displacement was in steps of $150 \mu \mathrm{m}$ $\overline{\left(0.6 \mathrm{~m}^{-1}\right)}$ and the fixed points were at a distance of

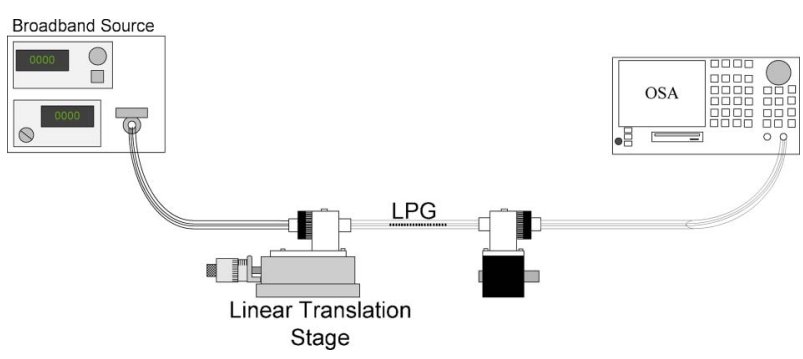

Fig. 4. Experimental setup for strain, torsion, bend, and refractive-index measurements.

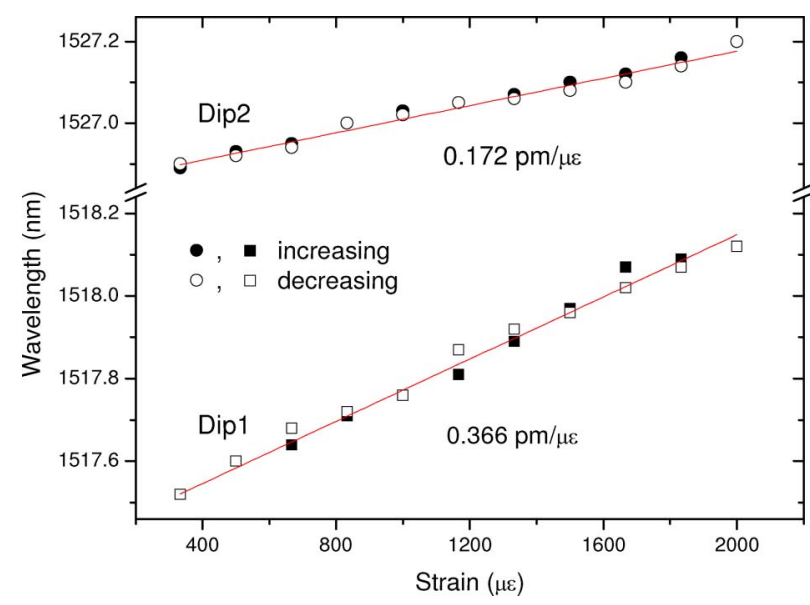

Fig. 5. (Color online) Dependence on strain of the resonance wavelengths of Dip1 and Dip2.

$20 \mathrm{~cm}$. It should be noted that the relation between displacement $d$ and curvature $C$ is nonlinear [10]:

$$
C=\frac{1}{R}=\frac{2 h}{h^{2}+\left[\frac{L_{0}-d}{2}\right]^{2}},
$$

where $h$ is the sagging of the fiber from the straight horizontal position, $L_{0}$ is the initial distance between the edges of the two fiber holders, and $R$ is the curvature radius. When the fiber is bent the structure geometry becomes curved and the refractive index is inhomogeneously changed due to the photoelastic effect. The bending of an initially straight fiber with curvature radius $R$ generates a strain $\varepsilon_{z}=x / R$ oriented parallel to the optical axis. This strain causes an index change for the $x$ - and $y$-polarization states that is equal for silica fiber to $\Delta n=-0.31 x / R$. The antisymmetric index change in a bent fiber results in modification of the cladding-mode profiles. The spectra exhibit shifts of LPG resonances in wavelength and changes in depth, and the notches frequently appear to split into two as a result of degeneracy breaking [11].

Figure 7 shows the grating spectra for a straight position and for bending values up to $2.1 \mathrm{~m}^{-1}$. It is observed that Dip1 and Dip2 shift toward longer wavelengths, with the shift being larger for Dip2. The amplitude of Dip2 increases when increased bending increases to $2.1 \mathrm{~m}^{-1}$; the amplitude of Dip1 decreases (resonance Dip1 was initially overcoupled). For bending curvatures higher than

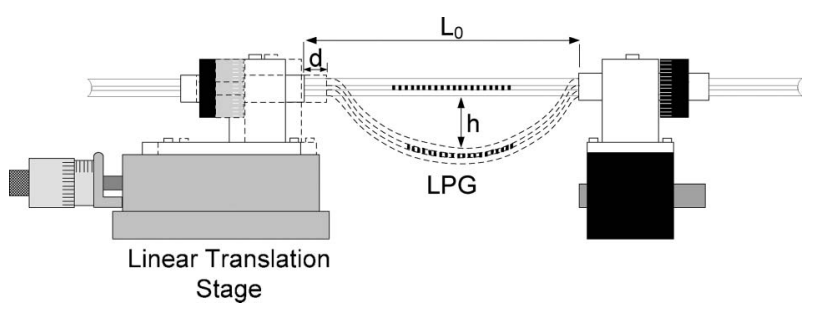

Fig. 6. Close-up view of the setup used for bending measurements. 


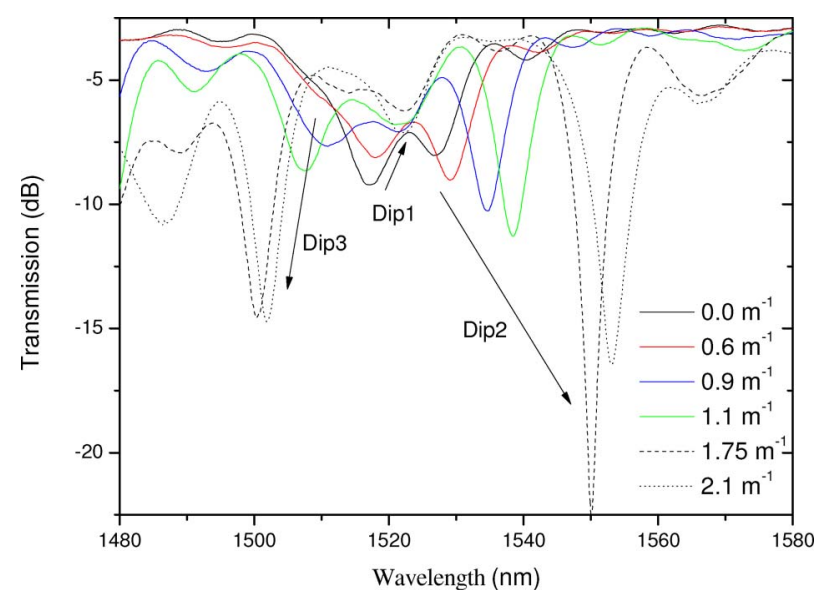

Fig. 7. (Color online) Grating spectra for bending curvatures up to $2.1 \mathrm{~m}^{-1}$.

$0.9 \mathrm{~m}^{-1}$, a third dip (Dip3) appears. This dip shifts toward shorter wavelengths and its amplitude increases when bending increases up to $2.1 \mathrm{~m}^{-1}$. Such behavior was observed in [11], where the effect of bending on long-period fiber gratings was studied. As shown in Fig. 8, the resonances (Dip1/Dip2 and Dip3) shift in opposite directions for bending values up to $\sim 2 \mathrm{~m}^{-1}$. The bending sensitivities of the resonances are approximately 2,17 , and, $-12 \mathrm{~nm} / \mathrm{m}^{-1}$ for Dip1, Dip2, and Dip3, respectively. For curvatures larger than $3.0 \mathrm{~m}^{-1}$ all the resonances move toward shorter wavelengths, the sensitivity being $-10,-25$, and $-5 \mathrm{~nm} / \mathrm{m}^{-1}$, respectively. Curvature measurements were also performed for different bending directions. The fiber was rotated in steps of $90^{\circ}$ and the measurements were repeated; similar results were obtained.

For torsion measurements, the fiber with the LPG was passed through the axes of two graduated disks. One of the disks is fixed and the other can be rotated clockwise or counterclockwise. To study the response of the grating to torsion, one end of the fiber was stationary, the other end was rotated in steps of $90^{\circ}$, and the distance between fixed points was set to $30 \mathrm{~cm}$.

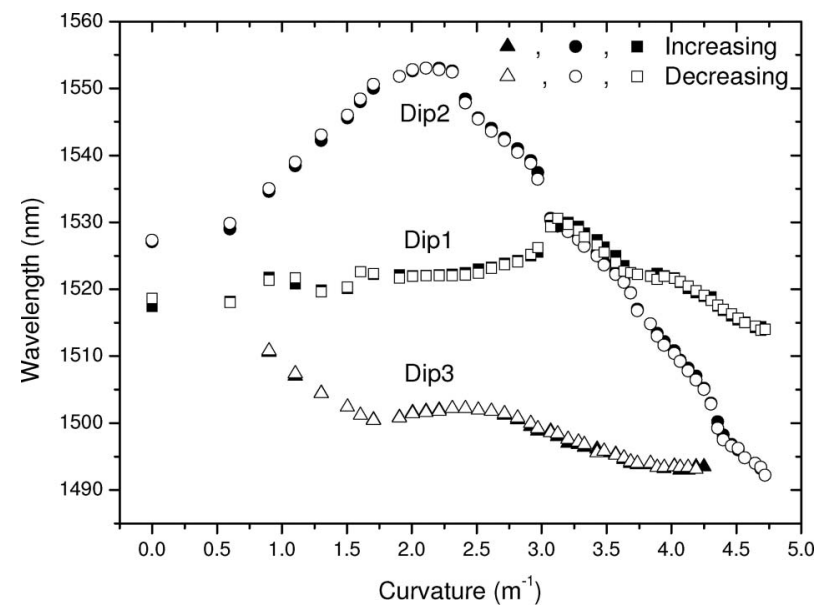

Fig. 8. Dependence on bending of the resonance wavelengths of Dip1, Dip2, and Dip3.
Thus the twisting step was equal to $\sim 5 \mathrm{rad} / \mathrm{m}$. As shown in Fig. 9, when the fiber is twisted, the position of the resonance at longer wavelengths fluctuates around the wavelength of $1527.0 \mathrm{~nm}$, whereas the wavelength of the resonance at shorter wavelengths increased almost linearly with torsion $[24.5 \mathrm{pm} /(\mathrm{rad} / \mathrm{m})]$. The behavior of arc-induced gratings under torsion [12] is not fully understood, but it might be similar to the behavior of gratings induced by $\mathrm{CO}_{2}$ laser radiation [13]. On the other hand, gratings induced by UV radiation [14], mechanical [15], and corrugated gratings [16] behave differently: the resonances shift toward shorter wavelengths independent of the direction of rotation. The mechanism of wavelength shift in twisted LPGs might be related to photoelastic effects induced by shear deformation in the fiber [17]. In this case, the mode self-coupling that is responsible for the wavelength shift is determined by the longitudinal electric field component. For large twist rates, this self-coupling might lead to resonance splitting.

Finally, we studied the response of the LPG to changes in the external refractive index. Some part of the cladding mode field propagates outside the fiber cladding in the form of an evanescent field. Since this field propagates through the outer medium, the propagation constants of cladding modes are sensitive to its refractive index [18]. We carried out measurements with LPGs for external refractive index in the range from 1.33 to 1.43 . Two different situations were considered: one when the grating was straight and the other when it had a fixed curvature. The study of the sensitivity to changes in external index of refraction was performed by immersing the LPG in samples of water combined with various percentages of ethylene glycol. The refractive index of different samples was calibrated using an Abbe refractometer with the sodium D line ( $589 \mathrm{~nm})$. Figure 10 shows the measurement results when the LPG was straight. In this case, Dip1 and Dip2 move toward shorter wavelengths with the shifts of approximately $1 \mathrm{~nm}$ for both resonances when the refractive index changes

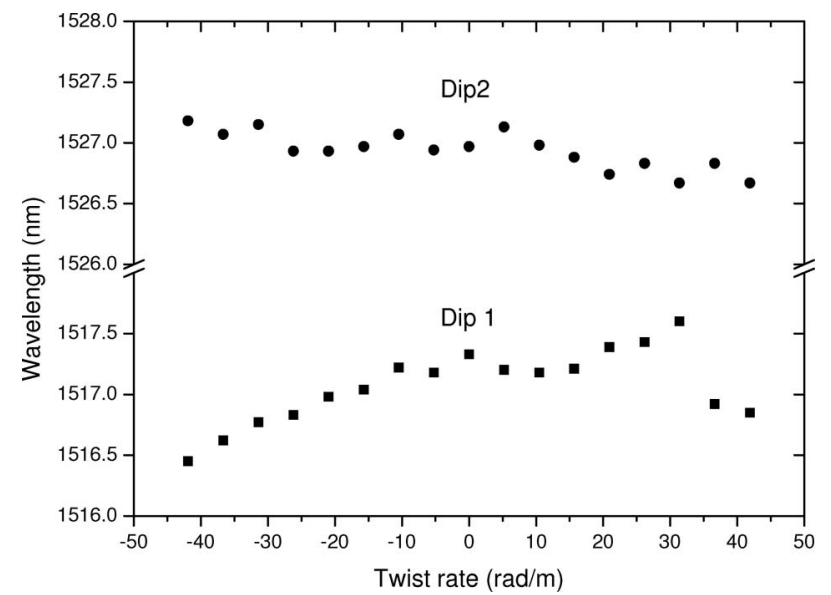

Fig. 9. Dependence on torsion of the resonance wavelengths of Dip1 and Dip2. 


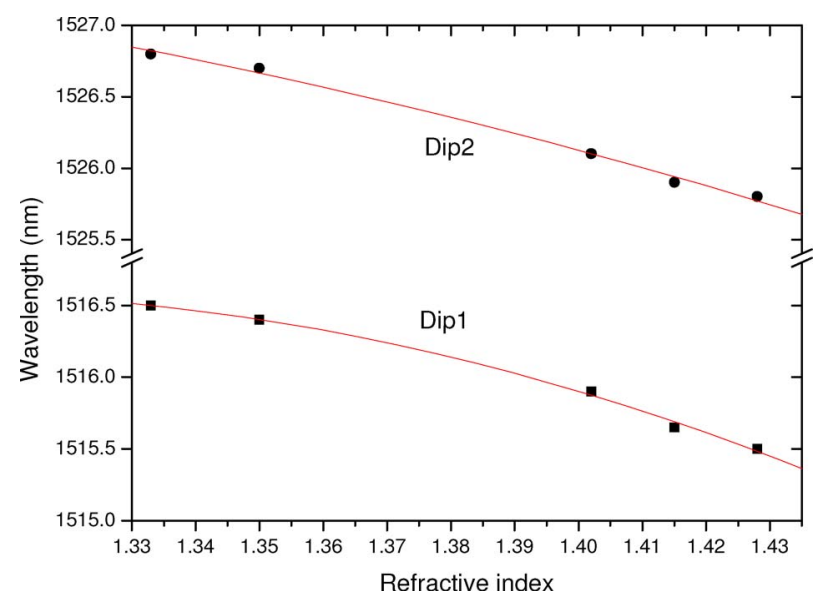

Fig. 10. (Color online) Shifts of the two resonance wavelengths of the LPG for different refractive indices of the external medium when the fiber is straight.

from 1.333 to 1.428 . This result is similar to that obtained by Khaliq et al. in [18].

Figure 11 shows the influence of the external refractive-index change on the resonance wavelengths for the curved LPG. A curvature of $2.1 \mathrm{~m}^{-1}$ was chosen to bend the LPG, which corresponds to the maximum separation between the two resonances (Dip1 and Dip2). For the same refractive-index change the shifts in wavelength were $0.8 \mathrm{~nm}$ for Dip 1 and $1.6 \mathrm{~nm}$ for Dip2, which differ from the shifts obtained for the straight LPG. Figure 11 also shows the wavelength dependence of Dip3 that exhibits the lowest sensitivity $(0.65 \mathrm{~nm})$ of the three dips.

Since the grating described here, like other LPGs, is sensitive to various physical parameters, during measurements of target parameters attention should be paid to keep other parameters fixed. For example, if we measure strain and temperature, an accidental bend or twist can affect the grating spectrum. Therefore, the fiber needs to be positioned in such a way that it cannot be bent or twisted. To protect the grating from the influence of external refractive index,

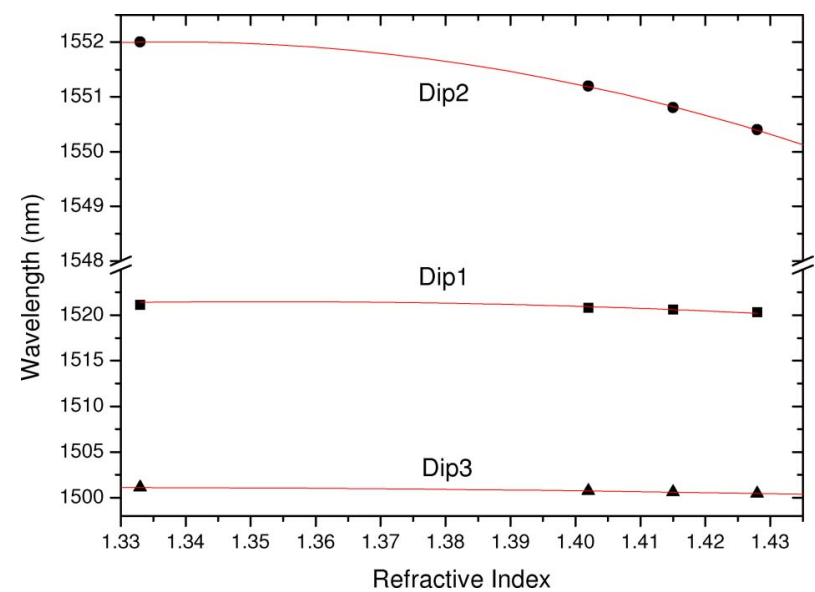

Fig. 11. (Color online) Shifts of the three resonance wavelengths of the LPG for different refractive indices of the external medium when the fiber has a curvature of $2.1 \mathrm{~m}^{-1}$. the fiber should be sealed. The temperature variations can be deduced from dual resonance measurements or can be eliminated by temperature stabilization.

\section{Conclusions}

We have characterized the response of a long-period fiber grating to changes in temperature, strain, bending, torsion, and external refractive index. The grating has been inscribed in a B/Ge co-doped fiber that exhibits two resonances resulting from coupling the core mode to cladding modes of different symmetries. It has been found that both resonances have similar temperature sensitivities $\left(\approx-288 \mathrm{pm} /{ }^{\circ} \mathrm{C}\right)$ and different strain, bending, and torsion sensitivities. The strain sensitivity of one resonance $(0.366 \mathrm{pm} / \mu \varepsilon)$ is twice as large as that of the other resonance $(0.172 \mathrm{pm} / \mu \varepsilon)$. When the fiber with the grating is curved, a new resonance appears and all the dips behave nonlinearly with increased curvature. When the grating is twisted, the wavelength of one resonance increases almost linearly $(24.5 \mathrm{pm} /(\mathrm{rad} / \mathrm{m}))$ with torsion whereas the wavelength of the other is essentially unaffected. The fiber grating is sensitive to refractive-index changes in the external medium: both resonances exhibit the same shift of $-1.0 \mathrm{~nm}$ when the refractive index increases from 1.333 to 1.428 . It has also been found that, when the fiber with the grating is curved, the sensitivities of all the dips differ $(0.8,1.6$, and $0.65 \mathrm{~nm})$. These results indicate the possibility of implementing temperature-compensated sensors for the measurement of one physical parameter such as strain, bend, or torsion.

This research was performed within the framework of the Fundação para a Ciência e a Tecnologia (FCT ) project "Evanescente," (POCTI/FIS/58877/ 2004). Paulo Caldas acknowledges the financial support of FCT (SFRH/BD/28653/2006). Oleg Ivanov acknowledges financial support from Russian Academy of Sciences (RAS) grants: support of basic research RAS Program N27 and Section of physical sciences RAS Program IV1.

\section{References}

1. G. Rego, O. Ivanov, and P. V. S. Marques, "Demonstration of coupling to symmetric and antisymmetric cladding modes in arc-induced long-period fiber gratings," Opt. Express 14, 9594-9599 (2006).

2. O. V. Ivanov and G. Rego, "Origin of coupling to antisymmetric modes in arc-induced long-period fiber gratings," Opt. Express 15, 13936-13941 (2007).

3. G. Rego and O. V. Ivanov, "Two types of resonances in longperiod gratings induced by arc discharges in B/Ge co-doped fibers," Opt. Lett. 32, 2984-2986 (2007).

4. S. W. James and R. P. Tatam, "Optical fibre long-period grating sensors: characteristics and application," Meas. Sci. Technol. 14, R49-R61 (2003).

5. V. Bhatia, D. Campbell, R. O. Claus, and A. M. Vengsarkar, "Simultaneous strain and temperature measurement with long-period gratings," Opt. Lett. 22, 648-650 (1997).

6. Y.-G. Han, S. Lee, C.-S. Kim, J. Kang, U.-C. Paek, and Y. Chung, "Simultaneous measurement of temperature and strain using 
dual long-period fiber gratings with controlled temperature and strain sensitivities," Opt. Express 11, 476-481 (2003).

7. G. Rego, R. Falate, O. Ivanov, and J. L. Santos, "Simultaneous temperature and strain measurements performed by a stepchanged arc-induced long-period fiber grating," Appl. Opt. 46, 1392-1396 (2007).

8. G. Rego, P. V. S. Marques, H. M. Salgado, and J. L. Santos, "Arc-induced long-period gratings," Fiber Integr. Opt. 24, 245-259 (2005).

9. O. V. Ivanov, "Coupling of hybrid modes in strained and heated fibers," Opt. Commun. 239, 311-321 (2004).

10. H. J. Patrick, C. C. Chang, and S. T. Vohra, "Long-period fibre gratings for structural bend sensing," Electron. Lett. 34, 1773-1775 (1998).

11. U. L. Block, V. Dangui, M. J. F. Digonnet, and M. M. Fejer, "Origin of apparent resonance mode splitting in bent long-period fiber gratings," J. Lightwave Technol. 24, 1027-1034 (2006).

12. J. M. Baptista, S. F. Santos, G. Rego, O. Frazão, and J. L. Santos, "Measurement of angular rotation using a long period fiber grating in a self-referenced fiber optic intensity sensor," in Proceedings of the 18th Annual Meeting of the IEEE Laser and Electro-Optics Society (IEEE, 2005), pp. 806-807.

13. Y. J. Rao, Y. P. Wang, Z. L. Ran, and T. Zhu, "Novel fiber-optic sensors based on long-period fiber gratings written by highfrequency $\mathrm{CO}_{2}$ laser pulses," J. Lightwave Technol. 21, 1320-1327 (2003).

14. Z. Zhang, W. Shi, K. Gao, and Z. Fang, "Twist characteristics of the ultraviolet-written long-period fiber gratings," Chin. Phys. Lett. 2, 565-567 (2004).

15. O. V. Ivanov, "Wavelength shift and split microbend longperiod of cladding mode resonances in fiber gratings under torsion," Opt. Commun. 232, 159-166 (2004).

16. O. V. Ivanov and L. A. Wang, "Wavelength shifts of claddingmode resonance in corrugated long-period fiber gratings under torsion," Appl. Opt. 42, 2264-2272 (2003).

17. O. V. Ivanov, "Propagation and coupling of hybrid modes in twisted fibers," J. Opt. Soc. Am. A 22, 716-723 (2005).

18. S. Khaliq, S. W. James, and R. P. Tatam, "Fiber-optic liquidlevel sensor using a long-period grating," Opt. Lett. 26, 1224-1226 (2001). 